\title{
SURFERS: BEWARE NOT ONLY OF SHARKS
}

E.Oñate ${ }^{1}$; J.L Ramos ${ }^{2}$; J. Igartua ${ }^{1}$,Amaia Camara1.
${ }^{1}$ Paediatric Intensive Care Unit, ${ }^{2}$ Department of Paediatric Surgery. Donostia University Hospital. San Sebastián .Spain.

BACKGROUND

- Shewanella spp. are saprophytic bacteria that are part of the marine microflora in warm climates and are rarely pathogenic.

However, Shewanella spp. infections are being increasingly reported and being implicated as an important and developing cause of maritime-associated disease.

\section{CASE REPORT}

* A previously healthy, fully immunized 9 year-old boy with fever and a swollen right thigh came to the emergency room. He otherwise had no significant medical history.

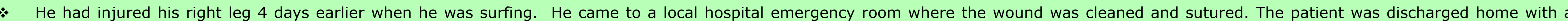

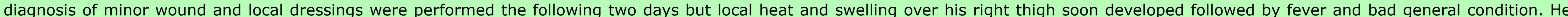
received cefuroxime two more days but finally he came again to a tertiary hospital.

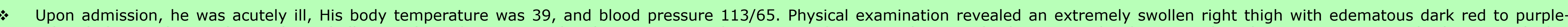

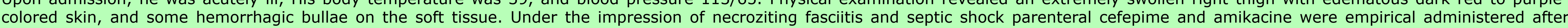
sampling blood for culture and an emergency operation was performed.

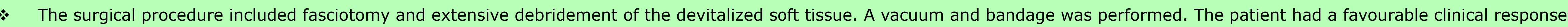

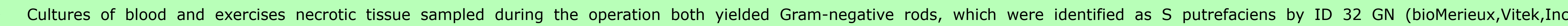
.Hazelwood,MO,USA)
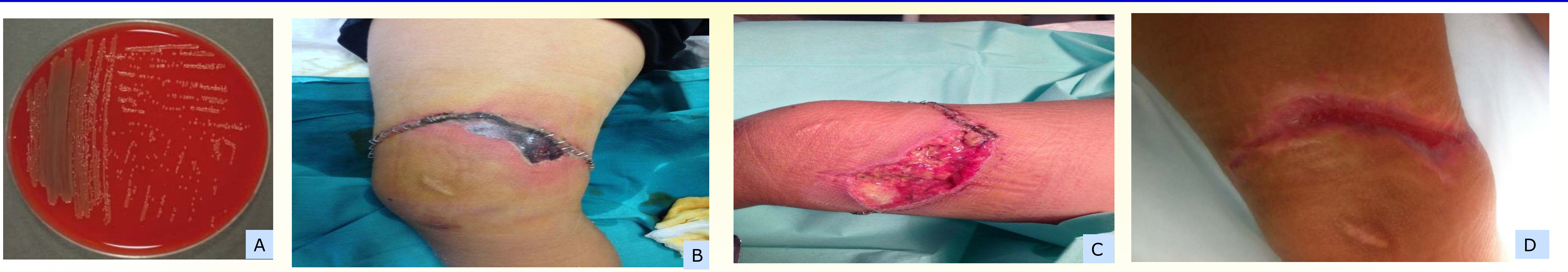

\begin{abstract}
A Shewanella culture
B Infection with necrotic plaque after mechanical suturing performed at different facility

C Result after surgical debridement

D FavoUrable outcome
\end{abstract}

\section{CONCLUSIONS}

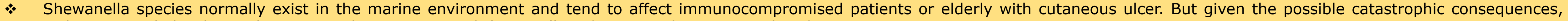
without a rapid abordaje, education on the prevention of shewanella soft tissue infections may be of importance. 